due to concentration changes brought about by the electrolysis. It was also shown that this non-linear relation could exist only if macroscopic space charges existed in the electrolyte.

The distribution of the macroscopic space charge along the column was calculated from the potential distribution. In every case it was apparent that the column of electrolyte contained four space-charge regions. Very near the cathode the charge was positive. The next quarter of the column contained negative space charge. Very near the anode the space charge was negative. The remainder of the column (about three-quarters of it) contained positive space charge. The largest space charge was observed within $1 \mathrm{~mm}$. from an anode; it required an excess of $2,000,000 \mathrm{Cu}^{++}$ions over the $\mathrm{SO}_{4}^{-}$ions per c.c., an excess of only 3 ions for every $10^{12} \mathrm{Cu}^{++}$ions originally present. The space charges in other parts of the column were very much smaller than this.

The distribution of the electric field-strength along the column of electrolyte was also calculated from the potential distribution. Although the magnitudes of the space charges were very small, they had very appreciable effects on the field-strengths. For ex. ample, the observed mean potential gradient in the explorable part of the $40-\mathrm{cm}$. long column (to within $1 \mathrm{~mm}$. of each electrode) was 16 per cent less than the average potential gradient $\left(\frac{8000}{40} \mathrm{mV} . / \mathrm{cm}\right.$.) in the cases of two solutions. Since the actual field-strengths are not constant and are so much different from those calculated from the potential difference between the electrodes and their distance apart, any directcurrent method for determining electrolytic conductivity must take space charge effects into account.

The eight electrolytes studjed were $0.0024 \mathrm{~N}$ solutions of copper, zinc and nickel sulphates and chlorides, zine acetate and aluminium sulphate. In each case the column of electrolyte was $40 \mathrm{~cm}$. long by $8 \mathrm{~cm}$. wide by $8 \mathrm{~cm}$. deep, and the electrodes were made of pure metal, the metal being that of the positive ions.

The results of this research force one to the conclusion that macroscopic space charges do exist in an electrolyte during electrolysis, and that this phenomenon is more complicated than it is in uni. formly ionized gases. It is expected that this newly discovered phenomenon will cause a re-examination of the theories concerning electrolysis.

A detailed account of this work will appear in the very near future in the Journal of Chemicul Physics.

William Schriever

Department of Physics,

University of Oklahoma,

Norman. Oklahoma.

$$
\text { Oct. } 29 .
$$

\section{Supersonic Vibration Potentials (Debye Effect) in Colloidal Solutions}

THE interesting results reported by Yeager, Bugosh, Hovorka and McCarthy ${ }^{1}$ with respect to the Debye effect in electrolytic solutions induce us to give an account of the results obtained by us in recent years in colloidal solutions.

The apparatus used was similar to that described before ${ }^{2}$, so we will give here only the main points of difference from the original apparatus. The radiofrequency generator consisted of a 100-watt Hartley oscillator and a push-pull amplifier stage utilizing two 500-watt tubes. The amplifier consisted of a high-frequency amplifier stage, converter and two intermediate-frequency amplifier stages; maximum amplification 700,000 . This amplification factor turned out to be much larger than necessary in our colloidal solutions of arsenic trisulphide (in our previous experiments we used silver iodide). It was reduced to 22,500 . The background noise was equivalent to \pm 3 \%

We tried to measure the velocity amplitude of the supersonic wave by measuring the acoustical pressure, by means of a hvdrostatic balance with a hollow prism ; but, like Yeager et al., we could not obtain satisfactory results.

Our colloidal solution of arsenic trisulphide contained $163 \mathrm{gm}$. of $\mathrm{As}_{2} \mathrm{~S}_{3}$ per litre; the electric conductivity was $73 \times 10^{-5} \Omega^{-1} \mathrm{~cm}^{-1}$, the viscosity $\eta=0.013$ c.G.s.

The Debye effect was quite large in this solution; the potentials measured in the solution varied from $45 \mathrm{~V}$. maximum to $12 \mathrm{~V}$. minimum, which yields, after division by 22,500, an amplitude for the supersonic vibration potential of $33.000 / 22,500=1.47 \mathrm{mV}$.

For an (estimated) velocity amplitude $v_{0}=$ $2.75 \mathrm{~cm}$. $/ \mathrm{sec}$., and a $\zeta$-potential of $100 \mathrm{mV}$., our simple formula ${ }^{2}$

$$
\Phi_{0}=\frac{m n D \zeta v_{0} g}{4 \pi r \sigma}
$$

leads to $7 \mathrm{mV}$; therefore, the discrepancy between theoretical and experimental values, reported in our previous note (factor 100), is less serious now.

$$
\text { A. J. RutGers }
$$$$
\text { J. VIDTS }
$$

Laboratory for Physical Chemistry, University of Ghent. Sept. 5.

1 Yeager, E., et al., J. Chem. Phys., 17, 411 (1949).

'Rutgers, A. J., Nature, 157, 74 (1946).

\section{Double Orbit in the 30-MeV. Synchrotron}

AN examination of the shape of the gamma-ray output pulse of the Malvern 30-MeV. synchrotron ${ }^{1}$ shows two distinct pulses, both when the machine is working as a synchrotron and as a betatron. When the machine is working as a synchrotron, both pulses occur after the radio-frequency is switched off, one at the expected time, the other much sooner.

The existence of the earlier (or spurious) pulse may perhaps be explained in terms of a resonance phe. nomenon (discussed by Courant ${ }^{2}$ ) between the second harmonic of the betatron oscillation frequency and the angular rotational frequency.

The equation for radial oscillations about the equilibrium orbit of a betatron to the first order is

$$
\ddot{p}+(1-n) \omega^{2} p=0,
$$

where $p$ is the displacement of the electron, $\omega$ is the angular rotational frequency and $n$ is $-d(\log H) / d(\log r)$, $H$ being the axial field at a radius $r$. Thus when $n=0.75$, the oscillation frequency is equal to half the rotational frequency, since $(1-n)^{1 / 2} \omega=0.5 \omega$.

If $n$ is nearly equal to 0.75 , the position of maximum $\rho$ will slowly precess. Now equation ( 1 ) is only a first-order equation, but in general there are small terms also in $p^{2}$ and $\dot{p}^{2}$, which make the solution of equation (1) a complicated function, in which the frequency is dependent on the amplitude. The 